\title{
Brane with Transverse Rotation and Background Fields: Boundary State and Tachyon Condensation
}

\author{
Davoud Kamani \\ Physics Department, Amirkabir University of Technology (Tehran Polytechnic) \\ P.O.Box: 15875-4413, Tehran, Iran \\ e-mail:kamani@aut.ac.ir
}

\begin{abstract}
The boundary state corresponding to the $\mathrm{D} p$-brane with a transverse rotation in the presence of the Kalb-Ramond and tachyon background fields and a $U(1)$ internal field will be constructed. We shall investigate effects of the open string tachyon condensation on this brane via its boundary state. We demonstrate that the background fields and transverse rotation cannot protect the brane against the collapse. Our calculations are in the context of the bosonic string theory.
\end{abstract}

PACS numbers: 11.25.-w; 11.25.Uv

Keywords: Rotating brane; Background fields; Boundary state; Tachyon condensation. 


\section{Introduction}

Some significant and important steps have been made to introduce the D-branes as essential objects in the string theory [1, 2]. One of the main problem of the D-branes is their stability. The fate of an unstable D-brane can be investigated via the dynamics of the open string tachyon, i.e., the tachyon condensation process [3]. An unstable D-brane usually decays to another lower dimensional unstable D-brane as an intermediate state [4, 5, 6]. This intercurrent state eventually collapses to the closed string vacuum or decays to a lower dimensional stable configuration. There are various trusty approaches for studying these concepts, e.g.,: string field theory [7, 8], the first quantized string theory [3, 9, 10, the renormalization group flow method [11, 12, 13], and the boundary string field theory [5, 7, 8, 14].

On the other hand, we have the boundary state formalism for describing the D-branes [15] - [37]. A boundary state prominently encodes all properties of its corresponding Dbrane, and is a source for emitting all closed string states. Thus, this adequate state can be used to study the time evolution of the brane during the tachyon condensation process [24] - 28]. Note that the rolling tachyon has a boundary state description which is valid during the finite time. Therefore, after elapsing this time the energy of the system will be completely dissipated into the bulk [25, 26].

Among the various D-branes the dynamical-dressed branes motivated us to examine their behaviors under the tachyon condensation experience. This stimulation is due to the background fields and dynamics of such branes. Thus, in this paper we shall consider a single $\mathrm{D} p$-brane with a transverse rotation, which has been dressed with the KalbRamond field, a $U(1)$ gauge potential and an open string tachyon field. The boundary state, associated with this $\mathrm{D} p$-brane, enables us to study the response of it in conflicting with the tachyon condensation phenomenon. We shall observe that the rotation of the brane and its field-dressing do not induce a resistance to protect it against the collapse. That is, the dimensional reduction of the brane will drastically occur.

This paper is organized as follows. In Sec. 2, the boundary state, corresponding to a rotating $\mathrm{D} p$-brane with the foregoing background fields, will be constructed. In Sec.

3, evolution of this $\mathrm{D} p$-brane under the condensation of the open string tachyon will be investigated. Section 4 is devoted to the conclusions. 


\section{The boundary state corresponding to our dynamical- dressed $\mathbf{D} p$-brane}

We begin with the following closed string action

$$
\begin{aligned}
S= & -\frac{1}{4 \pi \alpha^{\prime}} \int_{\Sigma} \mathrm{d}^{2} \sigma\left(\sqrt{-h} h^{a b} g_{\mu \nu} \partial_{a} X^{\mu} \partial_{b} X^{\nu}+\epsilon^{a b} B_{\mu \nu} \partial_{a} X^{\mu} \partial_{b} X^{\nu}\right) \\
& +\frac{1}{2 \pi \alpha^{\prime}} \int_{\partial \Sigma} \mathrm{d} \sigma\left(A_{\alpha} \partial_{\sigma} X^{\alpha}+T^{2}\left(X^{\alpha}\right)\right),
\end{aligned}
$$

where $\Sigma$ is the worldsheet of a closed string which is emitted by a static D $p$-brane, and $\partial \Sigma$ is the boundary of it. The coordinates $\left\{x^{\alpha} \mid \alpha=0,1, \cdots, p\right\}$ specify the directions which are along the worldvolume of this brane, and the set $\left\{x^{i} \mid i=p+1, \cdots, d-1\right\}$ will be used for the perpendicular directions to it. The field $A_{\alpha}$ is a $U(1)$ gauge potential which lives in the worldvolume of the brane, and $T^{2}\left(X^{\alpha}\right)$ is the open string tachyon field.

In fact, the states of our tachyon field and the gauge potential belong to the open string spectrum. Thus, their corresponding fields obviously appear in the surface terms of the string action. This implies that these fields do not have any coupling with the worldsheet curvature. That is, the action (2.1) has the Weyl symmetry. Therefore, for the metric of the worldsheet we can choose the flat gauge $h_{a b}=\eta_{a b}=\operatorname{diag}(-1,1)$. Beside, the spacetime metric is chosen as $g_{\mu \nu}=\eta_{\mu \nu}=\operatorname{diag}(-1,1, \cdots, 1)$, and for the gauge field we select the gauge $A_{\alpha}=-\frac{1}{2} F_{\alpha \beta} X^{\beta}$ with the constant field strength $F_{\alpha \beta}$. In addition,

we apply the tachyon profile $T^{2}=-2 \pi i \alpha^{\prime} U_{\alpha \beta} X^{\alpha} X^{\beta}$ where the tachyon matrix $U_{\alpha \beta}$ is constant and symmetric.

The Kalb-Ramond field $B_{\mu \nu}$ also will be considered constant. This implies that the second term of the action is total derivative, i.e., it is a surface term. Since the equation of motion originates from the bulk part of the action, the constant $B$-field obviously does not contribute to the equation of motion.

Variation of the action defines the following equations for the boundary state, associated with the static $\mathrm{D} p$-brane with the background fields

$$
\begin{aligned}
& \left(\partial_{\tau} X^{\alpha}+\mathcal{F}^{\alpha}{ }_{\beta} \partial_{\sigma} X^{\beta}-B^{\alpha}{ }_{i} \partial_{\sigma} X^{i}-4 \pi i \alpha^{\prime} U^{\alpha}{ }_{\beta} X^{\beta}\right)_{\tau=0}|B\rangle_{(\text {static })}=0, \\
& \left(X^{i}-y^{i}\right)_{\tau=0}|B\rangle_{(\text {static })}=0
\end{aligned}
$$

where the transverse vector $y^{i}$ indicates the brane location, and $\mathcal{F}_{\alpha \beta}=F_{\alpha \beta}-B_{\alpha \beta}$ exhibits the total field strength. Making use of the second equation, the third term of the first 
equation vanishes.

Before rotating the brane we should remind the following facts. We know that string theory was started with the string action in the flat Minkowski background $\mathbb{R}^{1,25}$, and the flat hyperplanes (the D-branes) were discovered. However, in the string spectrum there exists a massless closed string state which prominently is corresponding to the fluctuations of the geometry [1]. Therefore, since the D-branes are sources of energymomentum tensors, the flatness of the background spacetime in the presence of them is a reliable approximation which has been widely applied to the various subjects of the string theory and branes. In our setup we considered the flat spacetime, accompanied by the constant Kalb-Ramond field and a zero dilaton field. These imply that our static $\mathrm{D} p$-brane represents a trivial solution of the supergravity equations. That is, in the first approximation this brane does not induce a curvature to the spacetime. Besides, it does not live in a non-flat background.

In fact, the rotation of the perpendicular coordinates to a brane worldvolume, which describes a spinning brane, deforms the metric of the background spacetime. However, imposing some other motions to the brane does not change the metric. For example, in the flat spacetime see the D-branes with transverse velocities [1, 21, 34, 35, 36], the D-branes with tangential rotations [37], and so on. In fact, in these examples the first approximation of the background metric has been manifestly applied. Our D-brane will rotate in a transverse plane to itself, thus, for a small angular velocity we have a quasistatic D-brane. Hence, similar to the foregoing examples, at least for such small rotations we can apply the first approximation of the metric. This elaborates that the equations of the boundary state, corresponding to the rotating brane with the transverse rotation, and also the equation of motion of an emitted closed string from the brane will be reliably written in the initial flat spacetime.

Now we impose a transverse rotation to the brane. Let $x^{i_{0}}$ be the horizontal axis and $x^{\alpha_{0}}$ (with $\alpha_{0} \neq 0$ ) be the vertical one. At the time $t=0$ the direction $x^{\alpha_{0}}$ is along the brane, and the direction $x^{i_{0}}$ is perpendicular to it. The brane is rotating, e.g. counterclockwise, with the constant angular velocity " $\omega$ ". The axis of the rotation is one of the normal directions to the plane $x^{i_{0}} x^{\alpha_{0}}$. The coordinate system $\left\{x^{\prime \mu}\right\}$ is stuck to the brane such that at each moment the planes $x^{i_{0}} x^{\alpha_{0}}$ and $x^{\prime i_{0}} x^{\prime \alpha_{0}}$ have common origin and 
they are coincident. Thus, we receive the following coordinate transformations

$$
\begin{aligned}
& x^{\prime i_{0}}=x^{i_{0}} \cos (\omega t)+x^{\alpha_{0}} \sin (\omega t), \\
& x^{\prime \alpha_{0}}=-x^{i_{0}} \sin (\omega t)+x^{\alpha_{0}} \cos (\omega t), \\
& x^{\prime \bar{\alpha}}=x^{\bar{\alpha}}, \\
& x^{\bar{i}}=x^{\bar{i}},
\end{aligned}
$$

where the new indices $\bar{\alpha}$ and $\bar{i}$ belong to the sets

$$
\begin{aligned}
& \bar{\alpha} \in\{0,1, \ldots, p\}-\left\{\alpha_{0}\right\}, \\
& \bar{i} \in\{p+1, \ldots, d-1\}-\left\{i_{0}\right\} .
\end{aligned}
$$

The rotating $\mathrm{D} p$-brane possesses the following boundary state equations

$$
\begin{aligned}
& {\left[\partial_{\tau} X^{\bar{\alpha}}+\mathcal{F}^{\bar{\alpha}}{ }_{\bar{\beta}} \partial_{\sigma} X^{\bar{\beta}}+\mathcal{F}_{\alpha_{0}}^{\bar{\alpha}} \cos (\omega t)\left(-\sin (\omega t) \partial_{\sigma} X^{i_{0}}+\cos (\omega t) \partial_{\sigma} X^{\alpha_{0}}\right)\right.} \\
& \left.-4 \pi i \alpha^{\prime} U^{\bar{\alpha}}{ }_{\bar{\beta}} X^{\bar{\beta}}-4 \pi i \alpha^{\prime} U_{\alpha_{0}}^{\bar{\alpha}} \cos (\omega t)\left(-X^{i_{0}} \sin (\omega t)+X^{\alpha_{0}} \cos (\omega t)\right)\right]_{\tau=0}|B(t)\rangle=0, \\
& {\left[\cos (\omega t) \partial_{\tau} X^{\alpha_{0}}-\sin (\omega t) \partial_{\tau} X^{i_{0}}+\mathcal{F}_{\bar{\beta}}^{\alpha_{0}} \cos (\omega t) \partial_{\sigma} X^{\bar{\beta}}-4 \pi i \alpha^{\prime} U_{\bar{\beta}}^{\alpha_{0}} \cos (\omega t) X^{\bar{\beta}}\right.} \\
& \left.-4 \pi i \alpha^{\prime} U_{\alpha_{0}}^{\alpha_{0}} \cos ^{2}(\omega t)\left(-X^{i_{0}} \sin (\omega t)+X^{\alpha_{0}} \cos (\omega t)\right)\right]_{\tau=0}|B(t)\rangle=0 \\
& {\left[X^{i_{0}} \cos (\omega t)+X^{\alpha_{0}} \sin (\omega t)\right]_{\tau=0}|B(t)\rangle=0} \\
& \left(X^{\bar{i}}-y^{\bar{i}}\right)_{\tau=0}|B(t)\rangle=0 .
\end{aligned}
$$

Note that the time variable " $t$ " is the center-of-mass part of the emitted closed string coordinate $X^{0}(\sigma, \tau)$, i.e. $t=x^{0}$. Therefore, the argument of the sine and cosine is " $\omega t$ " but not " $\omega X^{0}$ ". If we use $\omega=\mathrm{d} \theta / \mathrm{d} X^{0}(\sigma, \tau)$, instead of $\omega=\mathrm{d} \theta / \mathrm{d} x^{0}$, we obtain a nonconstant angular velocity $\omega(\sigma, \tau)$. In this case each point of the emitted closed string from the rotating brane possesses its own angular velocity, which is not consistent with the assumption of the constant angular velocity of the rotating brane.

Eqs. (2.4) can be rewritten in terms of the zero modes and oscillators of the closed 
string coordinates

$$
\begin{aligned}
& {\left[p^{\bar{\alpha}}-2 \pi i U^{\bar{\alpha}}{ }_{\bar{\beta}} x^{\bar{\beta}}-2 \pi i U_{\alpha_{0}}^{\bar{\alpha}} \cos (\omega t)\left(-x^{i_{0}} \sin (\omega t)+x^{\alpha_{0}} \cos (\omega t)\right)\right]|B(t)\rangle^{(0)}=0,} \\
& {\left[p^{\alpha_{0}} \cos (\omega t)-p^{i_{0}} \sin (\omega t)-2 \pi i U_{\bar{\beta}}^{\alpha_{0}} \cos (\omega t) x^{\bar{\beta}}\right.} \\
& \left.-2 \pi i U_{\alpha_{0}}^{\alpha_{0}} \cos ^{2}(\omega t)\left(-x^{i_{0}} \sin (\omega t)+x^{\alpha_{0}} \cos (\omega t)\right)\right]|B(t)\rangle^{(0)}=0, \\
& {\left[x^{i_{0}} \cos (\omega t)+x^{\alpha_{0}} \sin (\omega t)\right]|B(t)\rangle^{(0)}=0,} \\
& \left(x^{\bar{i}}-y^{\bar{i}}\right)|B(t)\rangle^{(0)}=0
\end{aligned}
$$

for the zero-mode part, and we have

$$
\begin{aligned}
& {\left[\alpha_{m}^{\bar{\alpha}}+\tilde{\alpha}_{-m}^{\bar{\alpha}}-\left(\mathcal{F}_{\bar{\beta}}^{\bar{\alpha}}-\frac{2 \pi \alpha^{\prime}}{m} U^{\bar{\alpha}}\right)\left(\alpha_{m}^{\bar{\beta}}-\tilde{\alpha}_{-m}^{\bar{\beta}}\right)\right.} \\
& +\left(\mathcal{F}_{\alpha_{0}}^{\bar{\alpha}}-\frac{2 \pi \alpha^{\prime}}{m} U_{\alpha_{0}}^{\bar{\alpha}}\right) \cos (\omega t)\left[\left(\alpha_{m}^{i_{0}}-\tilde{\alpha}_{-m}^{i_{0}}\right) \sin (\omega t)\right. \\
& \left.-\left(\alpha_{m}^{\alpha_{0}}-\tilde{\alpha}_{-m}^{\alpha_{0}}\right) \cos (\omega t)\right]|B(t)\rangle^{(\text {osc })}=0 \\
& {\left[\left(\alpha_{m}^{\alpha_{0}}+\tilde{\alpha}_{-m}^{\alpha_{0}}\right) \cos (\omega t)-\left(\alpha_{m}^{i_{0}}+\tilde{\alpha}_{-m}^{i_{0}}\right) \sin (\omega t)\right.} \\
& -\left(\mathcal{F}^{\alpha_{0}}-\frac{2 \pi \alpha^{\prime}}{m} U_{\bar{\beta}}^{\alpha_{0}}\right) \cos (\omega t)\left(\alpha_{m}^{\bar{\beta}}-\tilde{\alpha}_{-m}^{\bar{\beta}}\right) \\
& \left.-\frac{2 \pi \alpha^{\prime}}{m} U_{\alpha_{0}}^{\alpha_{0}} \cos { }^{2}(\omega t)\left[\left(\alpha_{m}^{i_{0}}-\tilde{\alpha}_{-m}^{i_{0}}\right) \sin (\omega t)-\left(\alpha_{m}^{\alpha_{0}}-\tilde{\alpha}_{-m}^{\alpha_{0}}\right) \cos (\omega t)\right]\right]|B(t)\rangle^{(\text {osc })}=0, \\
& {\left[\left(\alpha_{m}^{i_{0}}-\tilde{\alpha}_{-m}^{i_{0}}\right) \cos (\omega t)+\left(\alpha_{m}^{\alpha_{0}}-\tilde{\alpha}_{-m}^{\alpha_{0}}\right) \sin (\omega t)\right]|B(t)\rangle^{(\text {osc })}=0,} \\
& \left(\alpha_{m}^{\bar{i}}-\tilde{\alpha}_{-m}^{\bar{i}}\right)|B(t)\rangle^{(\text {osc })}=0,
\end{aligned}
$$

for the oscillating part, with $m \in \mathbb{Z}-\{0\}$. Note that we decomposed the boundary state to the zero-mode portion and the oscillating part, i.e., $|B(t)\rangle=|B(t)\rangle^{(0)} \otimes|B(t)\rangle^{(\text {osc })}$.

In fact, solving Eqs. (2.5) and (2.6) is very difficult. For simplification we impose the restriction $U_{\alpha \alpha_{0}}=0$, or equivalently $U_{\bar{\alpha} \alpha_{0}}=U_{\alpha_{0} \alpha_{0}}=0$. Therefore, the solution of the 
zero-mode part of the boundary state is given by

$$
\begin{aligned}
|B(t)\rangle^{(0)} & =\frac{1}{\sqrt{\operatorname{det} \tilde{U}}} \int_{-\infty}^{\infty} \exp \left[-\frac{1}{4 \pi} \sum_{\bar{\alpha}}\left(\tilde{U}^{-1}\right)_{\bar{\alpha} \bar{\alpha}}\left(p^{\bar{\alpha}}\right)^{2}\right. \\
& \left.-\frac{1}{2 \pi} \sum_{\bar{\alpha} \neq \bar{\beta}}\left(\tilde{U}^{-1}\right)_{\bar{\alpha} \bar{\beta}} p^{\bar{\alpha}} p^{\bar{\beta}}\right]\left(\prod_{\bar{\alpha}}\left|p^{\bar{\alpha}}\right\rangle \mathrm{d} p^{\bar{\alpha}}\right) \\
& \times \delta\left[x^{i_{0}} \cos (\omega t)+x^{\alpha_{0}} \sin (\omega t)\right] \\
& \times \prod_{\bar{i}} \delta\left(x^{\bar{i}}-y^{\bar{i}}\right)\left|p^{\bar{i}}=0\right\rangle \otimes\left|p^{i_{0}}=0\right\rangle \otimes\left|p^{\alpha_{0}}=0\right\rangle,
\end{aligned}
$$

where, according to the condition $U_{\alpha \alpha_{0}}=0$, the $p \times p$ symmetric matrix $\tilde{U}$ is defined by eliminating the $\alpha_{0}$ th column and $\alpha_{0}$ th row of the $(p+1) \times(p+1)$ tachyon matrix $U$. From the disk partition function we deduce the prefactor $1 / \sqrt{\operatorname{det} \tilde{U}}$ [29]. The exponential part of $|B(t)\rangle^{(0)}$, which is absent for the conventional boundary states, clearly is an effect of the tachyon field. We observe that the zero-mode part of the boundary state is independent of the total field strength and the parameter $\alpha^{\prime}$. This is due to the fact that we considered a non-compact brane. The compact case extremely contains these factors [22].

For solving Eqs. (2.6) we define the new oscillators

$$
\begin{gathered}
A_{m}=\alpha_{m}^{i_{0}} \cos (\omega t)+\alpha_{m}^{\alpha_{0}} \sin (\omega t), \\
\tilde{A}_{m}=\tilde{\alpha}_{m}^{i_{0}} \cos (\omega t)+\tilde{\alpha}_{m}^{\alpha_{0}} \sin (\omega t), \\
B_{m}=\alpha_{m}^{\alpha_{0}} \cos (\omega t)-\alpha_{m}^{i_{0}} \sin (\omega t), \\
\tilde{B}_{m}=\tilde{\alpha}_{m}^{\alpha_{0}} \cos (\omega t)-\tilde{\alpha}_{m}^{i_{0}} \sin (\omega t) .
\end{gathered}
$$

These oscillators possess the following nonzero commutators

$$
\left[A_{m}, A_{n}\right]=\left[\tilde{A}_{m}, \tilde{A}_{n}\right]=\left[B_{m}, B_{n}\right]=\left[\tilde{B}_{m}, \tilde{B}_{n}\right]=m \delta_{m+n, 0},
$$

and all other commutators among them vanish.

By applying the coherent state method, and after some heavy calculations, the oscillating part of the boundary state finds the feature

$$
\begin{aligned}
& |B(t)\rangle^{(\text {osc })}=\frac{T_{p}}{g_{s}} \prod_{n=1}^{\infty}\left[\operatorname{det}\left(1-\mathcal{F}^{\prime}(t)+\frac{2 \pi \alpha^{\prime}}{n} \bar{U}\right)\right]^{-1} \\
& \times \exp \left\{-\sum_{m=1}^{\infty} \frac{1}{m}\left[\alpha_{-m}^{\bar{\alpha}} Q_{(m) \bar{\alpha} \bar{\beta}} \tilde{\alpha}_{-m}^{\bar{\beta}}-\alpha_{-m}^{\bar{i}} \tilde{\alpha}_{-m}^{\bar{i}}-A_{-m} \tilde{A}_{-m}\right.\right. \\
& +\left(1+2\left(M_{m}^{-1}\right)^{\bar{\alpha}}{ }_{\bar{\beta}} \mathcal{F}^{\bar{\beta}}{ }_{\alpha_{0}} \mathcal{F}^{\alpha_{0}} \cos ^{2}(\omega t)\right) B_{-m} \tilde{B}_{-m} \\
& \left.\left.+2 \mathcal{F}^{\bar{\beta}}{ }_{\alpha_{0}} \cos (\omega t)\left(\left(M_{m}^{-1}\right)_{\bar{\alpha} \bar{\beta}} \alpha_{-m}^{\bar{\alpha}} \tilde{B}_{-m}-\left(M_{m}^{-1}\right)_{\bar{\beta} \bar{\alpha}} B_{-m} \tilde{\alpha}_{-m}^{\bar{\alpha}}\right)\right]\right\}|0\rangle(2.1
\end{aligned}
$$


where by putting the $\alpha_{0}$ th column and $\alpha_{0}$ th row of the tachyon matrix $U$ to zero the $(p+1) \times(p+1)$ matrix $\bar{U}$ is obtained. By multiplying the $\alpha_{0}$ th column and $\alpha_{0}$ th row of the field strength matrix $\mathcal{F}$ with $\cos (\omega t)$ we receive the matrix $\mathcal{F}^{\prime}(t)$. The other matrices are defined by

$$
\begin{aligned}
& Q_{(m) \bar{\alpha} \bar{\beta}}=\left(M_{m}^{-1} N_{m}\right)_{\bar{\alpha} \bar{\beta}}, \\
& M_{(m) \bar{\beta}}^{\bar{\alpha}}=\delta^{\bar{\alpha}}{ }_{\bar{\beta}}-\mathcal{F}^{\bar{\alpha}}{ }_{\bar{\beta}}+\frac{2 \pi \alpha^{\prime}}{m} U^{\bar{\alpha}}{ }_{\bar{\beta}}-\mathcal{F}_{\alpha_{0}}^{\bar{\alpha}} \mathcal{F}_{\bar{\beta}}^{\alpha_{0}} \cos ^{2}(\omega t), \\
& N_{(m) \bar{\beta}}^{\bar{\alpha}}=\delta^{\bar{\alpha}}{ }_{\bar{\beta}}+\mathcal{F}^{\bar{\alpha}}{ }_{\bar{\beta}}-\frac{2 \pi \alpha^{\prime}}{m} U^{\bar{\alpha}}{ }_{\bar{\beta}}+\mathcal{F}_{\alpha_{0}}^{\bar{\alpha}} \mathcal{F}_{\bar{\beta}}^{\alpha_{0}} \cos ^{2}(\omega t) .
\end{aligned}
$$

As we see these matrices depend on the mode number " $m$ " which is induced by the tachyon matrix. The normalization factor, i.e., the infinite product in the first line of Eq. (2.10), is originated by the disk partition function. The state (2.10) specifies that $A_{m}$ and $\tilde{A}_{m}$ are Dirichlet oscillators. Similarly, in the case $\mathcal{F}_{\alpha_{0} \bar{\alpha}}=0$ the variables $B_{m}$ and $\tilde{B}_{m}$ became Neumann oscillators.

In Eqs. (2.6) one can express the right-moving annihilation oscillators in terms of the left-moving creation oscillators. This obviously eventuates to the boundary state (2.10). However, in these equations it is possible to express the left-moving annihilation oscillators in terms of the right-moving creation oscillators. In this case, applying the coherent state method leads to another form for the boundary state of the oscillating part. Equality of these boundary states elaborates the following conditions

$$
\begin{aligned}
& M_{m} M_{m}^{\prime T}=N_{m} N_{m}^{\prime T}, \\
& 2\left(M_{m}^{-1}\right)_{\bar{\alpha} \bar{\beta}} \mathcal{F}_{\alpha_{0}}^{\bar{\beta}}=-\mathcal{F}_{\bar{\beta}}^{\alpha_{0}}\left(Q_{m}^{\prime}+\mathbf{1}\right)_{\bar{\alpha}}^{\bar{\beta}}, \\
& 2\left(N_{m}^{\prime-1}\right)_{\bar{\alpha} \bar{\beta}} \mathcal{F}_{\alpha_{0}}^{\bar{\beta}}=-\mathcal{F}_{\bar{\beta}}^{\alpha_{0}}\left(Q_{m}+\mathbf{1}\right)_{\bar{\alpha}}^{\bar{\beta}}, \\
& \left(M_{m}^{-1}\right)_{\bar{\alpha} \bar{\beta}} \mathcal{F}_{\alpha_{0}}^{\bar{\beta}}=\left(N_{m}^{\prime-1}\right)_{\bar{\alpha} \bar{\beta}} \mathcal{F}_{\alpha_{0}}^{\bar{\beta}},
\end{aligned}
$$

where the new matrices have the definitions

$$
\begin{aligned}
& Q_{(m) \bar{\alpha} \bar{\beta}}^{\prime}=\left(N_{m}^{\prime-1} M_{m}^{\prime}\right)_{\bar{\alpha} \bar{\beta}}, \\
& M_{(m) \bar{\beta}}^{\prime \bar{\alpha}}=\delta^{\bar{\alpha}}{ }_{\bar{\beta}}-\mathcal{F}^{\bar{\alpha}}{ }_{\bar{\beta}}-\frac{2 \pi \alpha^{\prime}}{m} U^{\bar{\alpha}}{ }_{\bar{\beta}}+\mathcal{F}_{\alpha_{0}}^{\bar{\alpha}} \mathcal{F}_{\bar{\beta}}^{\alpha_{0}} \cos ^{2}(\omega t), \\
& N_{(m) \bar{\beta}}^{\prime \bar{\alpha}}=\delta^{\bar{\alpha}}{ }_{\bar{\beta}}+\mathcal{F}^{\bar{\alpha}}{ }_{\bar{\beta}}+\frac{2 \pi \alpha^{\prime}}{m} U^{\bar{\alpha}}{ }_{\bar{\beta}}-\mathcal{F}_{\alpha_{0}}^{\bar{\alpha}} \mathcal{F}_{\bar{\beta}}^{\alpha_{0}} \cos ^{2}(\omega t) .
\end{aligned}
$$

In fact, by substituting the explicit forms of the matrices from Eqs. (2.11) and (2.13) into Eqs. (2.12) we see that the first, the second and the third equations of (2.12) are trivial 
identities. That is, they do not impose any relation among the parameters of our setup. For the odd values of the brane dimension " $p$ " the fourth equation is an identity, and for the even values of " $p$ " it only gives rise to the condition $\operatorname{det}\left(\mathcal{F}_{\bar{\alpha} \bar{\beta}}\right)=0$.

Note that the total boundary state includes a part which comes from the conformal ghosts. This portion manifestly is independent of the background fields and the brane rotation. Thus, it obviously is null under the tachyon condensation process. Hence, we shall not consider it.

\section{$3 \quad$ Effect of the tachyon condensation on our $\mathbf{D} p$-brane}

According to the Sen's papers [3], in the presence of the open string tachyonic field our knowledge about the vacua of the string theories, the fate of the D-branes, their instability, and so on, was improved. During the process of the tachyon condensation the brane drastically collapses, and finally we receive a collection of the closed strings. These imply that decadence of unstable objects is very important phenomenon. For example, these objects specify an approach to achieve the background independent formulation of string theory.

Since the boundary state is a source for emitting all quantum states of closed string, and accurately describes all properties of the corresponding brane, and comprises a specific normalization factor, it is a favorable and convenient tool for finding the treatment and behavior of a single D-brane under the experience of the tachyon condensation. Hence, in this section we shall use this adequate formalism.

For imposing the condensation on the tachyon field, some of the matrix elements of the tachyon matrix should be infinite. For this purpose let the system tend to the infrared fixed point via the limit $U_{p p} \rightarrow \infty$. This defines the tachyon condensation along the $x^{p}$-direction, where we assume $x^{\alpha_{0}} \neq x^{p}$. Now we should take the limit of the total boundary state to acquire the behavior of our dynamical-dressed $\mathrm{D} p$-brane under the tachyon condensation process.

At first we obtain the behavior of the zero-mode part of the boundary state, i.e., Eq. (2.7). Under the limit $U_{p p} \rightarrow \infty$ its prefactor transforms to

$$
\frac{1}{\sqrt{U_{p p} \operatorname{det} \tilde{\tilde{U}}}}
$$


where the $(p-1) \times(p-1)$ symmetric matrix $\tilde{\tilde{U}}$ is defined by eliminating the last and $\alpha_{0}$ th columns and also the last and $\alpha_{0}$ th rows of the tachyon matrix $U$. At the IR fixed point limit we have

$$
\lim _{U_{p p} \rightarrow \infty} \tilde{U}^{-1}=\left(\begin{array}{cc}
\tilde{\tilde{U}}^{-1} & \mathbf{0}_{(p-1) \times 1} \\
\mathbf{0}_{1 \times(p-1)} & 0
\end{array}\right) .
$$

Adding all these together we receive the limit

$$
\begin{aligned}
|\mathcal{B}(t)\rangle^{(0)} & =\frac{2 \pi}{\sqrt{U_{p p} \operatorname{det} \tilde{\tilde{U}}}} \int_{-\infty}^{\infty} \exp \left[-\frac{1}{4 \pi} \sum_{a}\left(\tilde{\tilde{U}}^{-1}\right)_{a a}\left(p^{a}\right)^{2}\right. \\
& \left.-\frac{1}{2 \pi} \sum_{a \neq b}\left(\tilde{\tilde{U}}^{-1}\right)_{a b} p^{a} p^{b}\right]\left(\prod_{a}\left|p^{a}\right\rangle \mathrm{d} p^{a}\right) \\
& \times \delta\left[x^{i_{0}} \cos (\omega t)+x^{\alpha_{0}} \sin (\omega t)\right] \\
& \times \prod_{\bar{i}} \delta\left(x^{\bar{i}}-y^{\bar{i}}\right)\left|p^{\bar{i}}=0\right\rangle \otimes \delta\left(x^{p}\right)\left|p^{p}=0\right\rangle \\
& \otimes\left|p^{i_{0}}=0\right\rangle \otimes\left|p^{\alpha_{0}}=0\right\rangle,
\end{aligned}
$$

where $a, b \in\{0,1, \ldots, p\}-\left\{\alpha_{0}, p\right\}$. Since the exponential factor has lost the momentum component $p^{p}$ we obtained the state $\sqrt{2 \pi}\left|x^{p}=0\right\rangle=2 \pi \delta\left(x^{p}\right)\left|p^{p}=0\right\rangle$. However, up to the factor $2 \pi / \sqrt{U_{p p}}$ the Eq. (3.2) accurately represents the zero-mode boundary state of a $\mathrm{D}(p-1)$-brane which is rotating inside the $x^{i_{0}} x^{\alpha_{0}}$-plane with the angular velocity $\omega$.

Now look at the oscillating part of the boundary state. By the method of the zeta function regularization we have $\prod_{n=1}^{\infty}(n \lambda) \rightarrow \sqrt{2 \pi / \lambda}$, and accordingly the prefactor of Eq. (2.10) possesses the limit

$$
\frac{T_{p-1} \sqrt{U_{p p}}}{g_{s}} \prod_{n=1}^{\infty}\left[\operatorname{det}\left(\mathbf{1}-\tilde{\mathcal{F}}^{\prime}+\frac{2 \pi \alpha^{\prime}}{n} \tilde{\bar{U}}\right)_{p \times p}\right]^{-1}
$$

where the profitable relation $2 \pi \sqrt{\alpha^{\prime}} T_{p}=T_{p-1}$ was used. For the forms of the $p \times p$ matrices $\tilde{\mathcal{F}}^{\prime}$ and $\tilde{\bar{U}}$, eliminate the last rows and last columns of the $(p+1) \times(p+1)$ matrices $\mathcal{F}^{\prime}$ and $\bar{U}$, respectively.

For calculating the limit of $M_{m}^{-1}$ we use

$$
\lim _{U_{p p} \rightarrow \infty} \operatorname{det} M_{m}=\frac{2 \pi \alpha^{\prime}}{m} U_{p p} \operatorname{det} M_{m}^{(p-1)}
$$

where by eliminating the last row and last column of $M_{m}$ the $(p-1) \times(p-1)$ matrix $M_{m}^{(p-1)}$ is acquired. Since the last row and last column of $M_{m}^{-1}$ contain the factor $1 / U_{p p}$ 
we receive

$$
\lim _{U_{p p} \rightarrow \infty} M_{m}^{-1}=\left(\begin{array}{cc}
\left(M_{m}^{(p-1)}\right)^{-1} & \mathbf{0}_{(p-1) \times 1} \\
\mathbf{0}_{1 \times(p-1)} & 0
\end{array}\right) .
$$

Beside, the limit of $Q_{m}=M_{m}^{-1} N_{m}$ finds the feature

$$
\lim _{U_{p p} \rightarrow \infty} Q_{m}=\left(\begin{array}{cc}
\left(M_{m}^{(p-1)}\right)^{-1} N_{m}^{(p-1)} & \mathbf{0}_{(p-1) \times 1} \\
\mathbf{0}_{1 \times(p-1)} & -1
\end{array}\right) .
$$

Note that the limit of the matrix $Q_{m}$ is not the product of the limits of $M_{m}^{-1}$ and $N_{m}$. After performing the product $M_{m}^{-1} N_{m}$ we have taken the limit of $Q_{m}$. The structures of the matrices $M_{m}^{(p-1)}, N_{m}^{(p-1)}$ and $Q_{m}^{(p-1)}=\left(M_{m}^{(p-1)}\right)^{-1} N_{m}^{(p-1)}$ are similar to the matrices of Eq. (2.11) in which $\bar{\alpha}$ and $\bar{\beta}$ must be replaced with the indices $a, b \in\{0,1, \ldots p\}-\left\{\alpha_{0}, p\right\}$.

Adding all these together, the effect of the tachyon condensation on the oscillating part of the boundary state is given by

$$
\begin{aligned}
|\mathcal{B}(t)\rangle^{(\mathrm{osc})} & =\frac{T_{p-1} \sqrt{U_{p p}}}{g_{s}} \prod_{n=1}^{\infty}\left[\operatorname{det}\left(\mathbf{1}-\tilde{\mathcal{F}}^{\prime}+\frac{2 \pi \alpha^{\prime}}{n} \tilde{\bar{U}}\right)_{p \times p}\right]^{-1} \\
& \times \exp \left\{-\sum_{m=1}^{\infty} \frac{1}{m}\left[\alpha_{-m}^{a}\left(Q_{(m)}^{(p-1)}\right)_{a b} \tilde{\alpha}_{-m}^{b}-\alpha_{-m}^{p} \tilde{\alpha}_{-m}^{p}-\alpha_{-m}^{\bar{i}} \tilde{\alpha}_{-m}^{\bar{i}}-A_{-m} \tilde{A}_{-m}\right.\right. \\
& +2 \mathcal{F}^{b}{ }_{\alpha_{0}} \cos (\omega t)\left(\left[\left(M_{m}^{(p-1)}\right)^{-1}\right]_{a b} \alpha_{-m}^{a} \tilde{B}_{-m}-\left[\left(M_{m}^{(p-1)}\right)^{-1}\right]_{b a} B_{-m} \tilde{\alpha}_{-m}^{a}\right) \\
& \left.\left.+\left(1+2\left[\left(M_{m}^{(p-1)}\right)^{-1}\right]_{b}^{a} \mathcal{F}_{\alpha_{0}}^{b} \mathcal{F}_{a}^{\alpha_{0}} \cos ^{2}(\omega t)\right) B_{-m} \tilde{B}_{-m}\right]\right\}|0\rangle .
\end{aligned}
$$

As expected, the sign of the operator $\alpha_{-m}^{p} \tilde{\alpha}_{-m}^{p}$ has changed, i.e., the previous Neumann direction $x^{p}$ has been transformed to a Dirichlet direction. By comparing this equation with Eq. (2.10) we observe that, up to the factor $\sqrt{U_{p p}}$, Eq. (3.7) manifestly describes the oscillating part of the boundary state which is corresponding to the $\mathrm{D}(p-1)$-brane.

For the total boundary state at the IR fixed point the extra factors $1 / \sqrt{U_{p p}}$ and $\sqrt{U_{p p}}$ of Eqs. (3.2) and (3.7) exactly cancel each other. Similar cancellation between the zero-mode portion and the oscillating part also occurs in the D-D systems [8, 14, 38, However, according to the product of the states (3.2) and (3.7) we have proved that, during the tachyon condensation process, the transverse rotation and background fields cannot protect the brane against the collapse. That is, the unstable $\mathrm{D} p$-brane lost its $x^{p}$-direction and conveniently reduced to a $\mathrm{D}(p-1)$-brane. The resulted brane is rotating inside the 
$x^{i_{0}} x^{\alpha_{0}}$-plane with the same frequency " $\omega$ ". The delta functions of Eq. (3.2) prominently clarify that this $\mathrm{D}(p-1)$-brane has been localized at the position $x^{p}=0, x^{\bar{i}}=y^{\bar{i}}$, and its configuration at the times $t \in\left\{\frac{2 \pi n}{\omega} \mid n \in \mathbb{Z}\right\}$ is along the directions $\left\{x^{1}, x^{2}, \ldots, x^{p-1}\right\}$.

\section{Conclusions}

In the framework of the bosonic string theory we constructed a profitable boundary state, associated with a dynamical $\mathrm{D} p$-brane with a transverse rotation, in the presence of the anti-symmetric tensor field $B_{\mu \nu}$, a $U(1)$ internal gauge potential and a tachyonic field of the open string spectrum. Though we imposed a uniform rotation to the brane but the time dependence of the corresponding boundary state is very intricate. Besides, the rotational dynamics induced the deformed versions of the tachyon matrix and total field strength to the boundary state.

We investigated the effects of the tachyon condensation on the foregoing $\mathrm{D} p$-brane through its boundary state. We demonstrated that at the infrared fixed point the background fields, accompanied by the transverse rotation of the brane, cannot prevent the unstable brane against the collapse. Therefore, the tachyon condensation was eventually terminated by the dimensional reduction of the brane. The resulted $\mathrm{D}(p-1)$-brane possessed the same angular frequency as the previous one. Presence of the remaining tachyon field implies that the subsequent brane also is an unstable object, and at the IR fixed point will be collapsed.

\section{References}

[1] J. Polchinski, "String Theory", (Cambridge University Press, Cambridge, 1998), Volumes I and II; C.V. Johnson, "D-Branes", (Cambridge University Press, Cambridge, 2003).

[2] J. Polchinski, Phys. Rev. Lett. 75 (1995) 4724.

[3] A. Sen, Int. J. Mod. Phys. A 14 (1999) 4061; Int. J. Mod. Phys. A 20 (2005) 5513; JHEP 9808 (1998) 010; JHEP 9808 (1998) 012; JHEP 9912 (1999) 027; JHEP 9809 (1998) 023; JHEP 9812 (1998) 021; JHEP 9910 (1999) 008. 
[4] K. Hashimoto, P.M. Ho and J.E. Wang, Mod. Phys. Lett. A 20 (2005) 79.

[5] D. Kutasov, M. Marino and G. Moore, JHEP 0010 (2000) 045.

[6] T. Lee, Phys. Rev. D 64 (2001) 106004; Phys. Lett. B 520 (2001) 385.

[7] E. Witten, Phys. Rev. D 47 (1993) 3405; Phys. Rev. D 46 (1992) 5467; Nucl. Phys. B 268 (1986) 253.

[8] P. Kraus and F. Larsen, Phys. Rev. D 63 (2001) 106004.

[9] E. Witten, JHEP 12 (1998) 019.

[10] O. Bergman and M. R. Gaberdiel, Phys. Lett. B 441 (1998) 133.

[11] J. A. Harvey, D. Kutasov and E. J. Martinec, arXiv:hep-th/0003101.

[12] S. Dasgupta and T. Dasgupta, arXiv:hep-th/0010247.

[13] A. A. Gerasimov and S. L. Shatashvili, JHEP 10 (2000) 034.

[14] S. L. Shatashvili, Phys. Lett. B 311 (1993) 83; T. Takayanagi, S. Terashima and T. Uesugi, JHEP 03 (2001) 019.

[15] M.B. Green and M. Gutperle, Nucl. Phys. B 476 (1996) 484.

[16] C. Schmidhuber, Nucl. Phys. B 467 (1996) 146. M.B. Green and P. Wai, Nucl. Phys. B 431 (1994) 131.

[17] M. Billo, P. Di Vecchia, M. Frau, A. Lerda, I. Pesando, R. Russo and S. Sciuto, Nucl. Phys. B 526 (1998) 199.

[18] P. Di Vecchia, M. Frau, I. Pesando, S. Sciuto, A. Lerda and R. Russo, Nucl. Phys. B 507 (1997) 259.

[19] F. Hussain, R. Iengo and C. Nunez, Nucl. Phys. B 497 (1997) 205.

[20] O. Bergman, M. Gaberdiel and G. Lifschytz, Nucl. Phys. B 509 (1998) 194.

[21] M. Billo, P. Di Vecchia and D. Cangemi, Phys. Lett. B 400 (1997) 63. 
[22] H. Arfaei and D. Kamani, Phys. Lett. B 452 (1999) 54, arXiv:hep-th/9909167; Nucl. Phys. B 561 (1999) 57, arXiv:hep-th/9911146; Phys. Lett. B 475 (2000) 39-45, arXiv:hep-th/9909079.

[23] D. Kamani, Phys. Lett. B 487 (2000) 187-191, arXiv:hep-th/0010019; Europhys. Lett. 57 (2002) 672-676, arXiv:hep-th/0112153; Nucl. Phys. B 601 (2001) 149-168, arXiv:hep-th/0104089; Mod. Phys. Lett. A 15 (2000) 1655-1664, arXiv:hep-th/9910043; F. Safarzadeh-Maleki and D. Kamani, Phys. Rev. D 90, 107902 (2014), arXiv:1410.4948 [hep-th]; M. Saidy-Sarjoubi and D. Kamani, Phys. Rev. D 92, 046003 (2015), arXiv:1508.02084 [hep-th]; E. Maghsoodi and D. Kamani, Nucl. Phys. B 922 (2017) 280, arXiv:1707.08383 [hep-th].

[24] M. Naka, T. Takayanagi and T. Uesugi, JHEP 0006 (2000) 007.

[25] T. Okuda and S. Sugimoto, Nucl. Phys. B 647 (2002) 101.

[26] F. Larsen, A. Naqvi and S. Terashima, JHEP 0302 (2003) 039.

[27] A. Sen, JHEP 0204 (2002) 048; JHEP 0207 (2002) 065.

[28] D. Kamani, Annals of Physics 354 (2015) 394-400, arXiv:1501.02453 [hep-th].

[29] C.G. Callan, C. Lovelace, C.R. Nappi and S.A. Yost, Nucl. Phys. B 308 (1988) 221-284; E.S. Fradkin and A.A. Tseytlin, Phys. Lett. B 163 (1985) 123.

[30] J. Dai, R.G. Leigh and J. Polchinski, Mod. Phys. Lett. A4 (1989) 2073; R.G. Leigh, Mod. Phys. Lett. A 4 (1989) 2767; S.J. Rey and S. Sugimoto, Phys. Rev. D 68 (2003) 026003; M. Frau, I. Pesando, S. Sciuto, A. Lerda and R. Russo, Phys. Lett. B 400 (1997) 52; M. Li, Nucl. Phys. B 460 (1996) 351.

[31] S. Gukov, I. R. Klebanov and A. M. Polyakov, Phys. Lett. B 423 (1998) 64.

[32] T. Kitao, N. Ohta and J. G. Zhou, Phys. Lett. B 428 (1998) 68.

[33] C. Bachas, Phys. Lett. B 374 (1996) 37.

[34] C.G. Callan and I.R. Klebanov, Nucl. Phys. B 465 (1996) 473.

[35] P. Di Vecchia and A. Liccardo, "D branes in string theory, II", YITP Proceedings Series No. 4 (Kyoto, Japan, 1999). 
[36] F. Hussain, R. Iengo, C. Nunez and C.A. Scrucca, Nucl. Phys. B 517 (1998) 92; Phys. Lett. B 409 (1997) 101.

[37] F. Safarzadeh-Maleki and D. Kamani, Phys. Rev. D 89, 026006 (2014), arXiv:1312.5489 [hep-th]; D. Kamani and E. Maghsoodi, Nucl. Phys. B 942 (2019) 381, arXiv:1904.08207 [hep-th].

[38] S.P. de Alwis, Phys. Lett. B 505 (2001) 215. 